\section{Pilares personalizados y siste- mas cerámicos con tecnología CAD-CAM en el manejo estético del sector anterior sobre restau- raciones implanto soportadas. Reportes de casos.}

Huamaní-Chavez A ${ }^{1}$, Balarezo-Razzeto JA², Matta-Morales $\mathrm{C}^{2}$, Ccahuana-Vásquez $\mathrm{V}^{3}$. Pilares personalizados y sistemas cerámicos con tecnología CAD-CAM en el manejo estético del sector anterior sobre restauraciones implanto soportadas. Reportes de casos. Rev Estomatol Herediana. 2012; 22(2):100-108

\section{RESUMEN}

En el presente reporte se describen 03 casos clínicos de rehabilitación sobre implantes unitarios en el sector anterior: 01 sist. Lifecore-Renova $(3,75 \mathrm{~mm} \emptyset, 13 \mathrm{~mm}$. longitud, conexión interna), 02 del sist. Conexão (5 mm Ø x $13 \mathrm{~mm}$. de longitud; $4,3 \mathrm{~mm} \emptyset \times 15 \mathrm{~mm}$. de longitud, conexión interna). En la etapa de provisionales, se realizó manejo de tejidos blandos a través de restauraciones atornilladas provisionales (6-8 meses). En la rehabilitación definitiva se rehabilitaron, mediante el uso de: un pilar anatómico personalizado de oro-paladio y dos metalo-cerámicos; la parte coronaria fue restaurada con una corona metal-free de zirconio sistema Everest, y dos del sistema E-Max CAD (disilicato de litio). Palabras clave: IMPLANTES DENTALES, PILARES PERSONALIZADOS, CAD-CAM.

Customized abutments and ceramic systems with technology CAD-CAM in the aesthetic management of the previous sector on restorations implant supported. Reports of cases.

\section{ABSTRACT}

The aim of the present article is to describe of 03 clinical cases of rehabilitation on unitary implants in the previous sector: 01 sist. Lifecore-Renova $(3.75 \mathrm{~mm} \varnothing, 13 \mathrm{~mm}$. length, internal connection), 02 of the sist.Conexão ( $5 \mathrm{~mm} \varnothing \times 13 \mathrm{~mm}$. of length; $4.3 \mathrm{~mm} \emptyset \times 15 \mathrm{~mm}$. of length, internal connection). In the stage of provisionals, there was realized managing of soft tissue across screwed provisional restorations (6-8 months). In the definitive rehabilitation they recovered, by means of the use of: anatomical customized abutments of gold - palladium (1) and metalo-ceramic (2); the coronary part was restored by crowns metal-free of zirconio system Everest (1), and 02 of the system E-Max CAD (disilicato of lithium)

KEY WORDS: dental implants, customized abutments, cad-cam.

\section{Alexis Huamaní Chávez' José Antonio Balarezo Razzeto² Carlos Matta Morales ${ }^{2}$ Vanessa Ccahuana Vásquez ${ }^{3}$}

${ }^{1}$ Residente del Programa de Especialización en Rehabilitación Oral. Docente de PreGrado y Post-Grado de Rehabilitación Oral Facultad de Estomatología, Universidad Peruana Cayetano Heredia. Lima, Perú.

2Docente del Departamento Académico de la Clínica Estomatológica, Facultad de Estomatología, Universidad Peruana Cayetano Heredia. Lima, Perú.

${ }^{3}$ Coordinadora del Area de Post-Grado de Implantología Oral, Facultad de Estomatología, Universidad Peruana Cayetano Heredia. Lima, Perú.

\section{Correspondencia}

Alexis Aníbal Huamaní Chávez

Av. Guardia Civil 394-San Isidro

Lima, Perú

Teléfono: 511-997298268

E-mail: alexanibalhch@hotmail.com

\section{Introducción}

El reemplazo de dientes unitarios con implantes dentales es un tratamiento dental de rutina en la actualidad. Muchos estudios muestran tasas de éxito de más del $89 \%$ para coronas implanto soportadas, con altos niveles de satisfacción de parte del operador y del paciente (1). En la mayoría de situaciones clínicas en las que se va a colocar implantes en la región anterior, el clínico se ve enfrentado a muchos desafíos, no sólo referente a los requerimientos estéticos por parte de los pacientes, si no también, a retos previos a la colocación del dispositivo, ejemplo: defectos óseos del tipo Seibert I (ancho), Seibert II (alto), Seibert III (alto + ancho), es- pacios protésicos reducidos, tejidos blandos alterados (poca presencia o ausencia de tejido queratinizado), etc.; situaciones que deben ser corregidas previas a la colocación del implante ó, posteriores al mismo (injertos óseos, injertos de tejido conectivo, etc.), para poder tener una adecuada previsibilidad del tratamiento en el futuro.

La llave para el éxito de la terapia con implantes en la región anterior, es una relación armoniosa entre la restauración implanto soportada y los dientes naturales remanentes. Para lograr este objetivo, un implante debe ser planificado conceptualmente y colocado como una extensión vertical de una restauración óptimamente visualizada, como es descrito por Garber y Belser en los 3 conceptos de "restauraciónmanejada en la planificación del tratamiento con implantes". Este enfoque envuelve una planificación de tratamiento en 3 dimensiones (3D), seguido por la colocación del implante en una posición que es óptima para ambas situaciones: la función y la estética. La evaluación inicial en 3D del sitio potencial, debe incluir una planificación para el aumento o preservación de los tejidos óseos y gingivales existentes alrededor del diente comprometido. Esto puede ser una parte integral del proceso, porque es conocido que la extracción es siempre seguido, por alguna resorción del hueso, con la concomitante recesión del 
tejido blando. Para lograr un resultado estético óptimo, los clínicos deben considerar un punto adicional importante, la 4ta dimensión (considerando biológicamente a la "4ta dimensión" como el tiempo de respuesta del tejido y la secuencia del tratamiento). Este adicional componente en la planificación del tratamiento incorpora la secuencia óptima de etapas o tiempos de (2):

\section{Extracción del diente}

2. Preservación del mejoramiento de los tejidos duros y blandos

3. Colocación del implante

4. Conexión del pilar

5. Modelado del tejido con un provisional y/o la modificación del pilar; y

6. La restauración definitiva (2)

Una vez colocado el implante y definida la restauración definitiva a ser utilizada (cementada o atornillada), se debe tener precaución en: elegir un material que cumpla los requerimientos de biocompatibilidad necesarios, que tenga propiedades mecánicas adecuadas, que se restablezca un adecuado perfil de emergencia (pilar y corona), y se determine en forma adecuada la distancia desde la plataforma del implante hasta el nivel del margen de la encía (cuff) del pilar (abutment), para determinar adecuadamente la profundidad del margen de la preparación del pilar sobre el cual descansará la futura corona.

En el presente artículo, se hace hincapié en la importancia del uso de restauraciones provisionales para realizar un adecuado manejo de tejidos blandos con el objetivo de restablecer un perfil de emergencia óptimo, y de la personalización de los pilares (restauración definitiva) para lograr resultados estéticos adecuados en el sector anterior; lo- grando así restauraciones que sean proporcionales a las piezas dentales contiguas tanto en forma, proporciones, color, textura, y una adecuada interrelación con los tejidos blandos y duros subyacentes (Score estético blanco y rosado) (3).

En el 2009, Gershenfeld propuso criterios de revisión con la combinación de puntajes de evaluación estéticos rosados y blancos, este índice fue diseñado para la evaluación objetiva de los resultados de las dimensiones estéticas de los implantes en dientes unitarios de la región anterior maxilar. Los puntajes estéticos rosados (PES) fueron modificados de los 7 parámetros de Fürhauser a $5(3,4)$ :

- la papila mesial,

- papila distal,

- curvatura facial de la mucosa,

- nivel de la mucosa facial,

- convexidad de la raíz/color del tejido blando, y textura del aspecto facial del sitio del implante.

El puntaje de evaluación estético blanco (WES) se enfoca en la parte visible de la corona del implante que surge o emerge de la mucosa peri implantaria y es basada en 5 parámetros, estos incluyen:

- forma general del diente;

- contorno y volumen de la corona clínica;

- color incluyendo el valor y el matiz;

- textura de la superficie y translucencia; y

- caracterización.

La restauración de los dientes naturales con coronas cerámicas provee a los pacientes de una mayor estética. En la actualidad se dispone de materiales cerámicos con propiedades mecánicas mejoradas, casi comparables a las restauraciones metal cerámicas, aparte de las propiedades ópticas y de biocompatibilidad ya conocidas (5).

Por razones estéticas, la demanda por restauraciones implanto retenidas compuestas de abutments cerámicos y coronas totalmente cerámicas se ha incrementado en el tiempo. Las cerámicas de óxido de zirconio son usadas para confeccionar los abutments para implantes dentales, ya que ellos han mejorado en la actualidad su resistencia y su compatibilidad con los tejidos blandos. Sin embargo, la combinación de un abutment cerámico y una corona totalmente cerámica mejora la transmisión de la luz a través del tejido peri implantario (6). De forma similar, cuando son usados abutments de cerámica para restaurar implantes unitarios, altos resultados estéticos pueden ser obtenidos, particularmente cuando se les compara a los abutments metálicos tradicionales. Incluso, aunque las cerámicas más fuertes tengan fuerzas de fractura inferiores comparada a sus contrapartes metálicas, sobre todo ante cargas oblicuas, ellas todavía muestran un funcionamiento clínico aceptable.

La tecnología CAD/CAM ha demostrado su capacidad de fabricar restauraciones protéticas con la calidad comparable a sus contrapartes fabricados con técnicas convencionales $(7,8)$.

Las posibilidades expandidas del uso de la tecnología CAD/CAM permiten la fabricación de dentaduras parciales removibles, sistemas de dientes de dentaduras completas con una articulación virtual, abutments cerámicos implanto soportados y coronas cerámicas implanto soportadas.

Sin embargo, a la actualidad, son pocas las publicaciones que 
hacen referencia del uso de la tecnología $\mathrm{CAD} / \mathrm{CAM}$ para fabricar abutments personalizados cerámicos con coronas cerámicas asociadas de bloques cerámicos feldespáticos pre-sinterizados. Realizar este procedimiento puede en teoría ofrecer el reemplazo en una sola cita de un diente perdido con una corona estética permanente totalmente cerámica implanto soportada (1).

Cuando tenemos dudas sobre que abutment utilizar en la región anterior para un caso unitario, varios factores deben ser considerados:

1. Visibilidad de la región (ejemplo: línea de la sonrisa alta o baja)

2. Biotipo gingival

3. Color de los dientes vecinos, y finalmente

4. Expectativas del paciente.

En situaciones de alta demanda estética los abutments cerámicos personalizados están indicados. En situaciones con tejidos blandos peri-implantarios delgados, los implantes personalizados cerámicos combinados con coronas totalmente cerámicos son los indicados. En casos con mucosa gruesa, el titanio puede ser usado como el material de abutment, combinado con coronas metal cerámicas. Para evitar dificultades que se relacionan con la eliminación del cemento, las restauraciones atornilladas pueden ser preferidas; sin embargo, el agujero de acceso del tornillo debería ser colocado palatal al borde incisal (8).

\section{Caso 1: Pilar oro-pálido}

El paciente tenía en el momento del tratamiento 25 años de edad, presentó como antecedente estomatológico la fractura de la tabla ósea y la avulsión de la pieza dentaria 2.1 (accidente) (Figura 1). Se realizaron injertos óseos y de tejido conectivo, previos a la colocación del implante. A los 4 meses se instaló el implante y posterior al proceso de oseointegración (4 meses), se expuso el mismo y se inició la etapa de manejo de tejidos blandos a través del uso de una prótesis provisional atornillada (pilar UCLA Temporary Sleeve, Hexed SDI 43110) con frente estético de dientes de acrílico (Vita Triostat, color 2M2, Vita) (Figuras 2 y 3). El tiempo del manejo de tejidos blandos con la provisional atornillada fue de $8 \mathrm{me}$ ses (6 meses de manipulación y 2 meses de estabilización) para tener la seguridad de una adecuada estabilidad de los tejidos blandos en el tiempo (Figuras 4,5,6,7 ,8 y 9).

En la etapa de rehabilitación definitiva se personalizó un pilar UCLA Gold/Plastic Combo SleeveLocking SDI (43006), según el perfil de emergencia logrado a través de la etapa de manejo de tejidos blandos con la restauración provisional (llave de silicona del perfil, forma y contorno de la restauración provisional); perfil que fue reproducido a través de una impresión personalizada, con técnica de cubeta abierta (ImplantImpression post SDI 43127) (Figuras 10, 11 y 12). El Pilar ya personalizado se revistió y se hizo el colado posterior con una aleación de oro-paladio, se realizó un pulido prolijo posterior (Figura 13). Se verificó el adecuado asentamiento del mismo en el implante (verificación radiográfica). Una vez determinada la forma final del pilar, se hizo el escaneado de los márgenes y el diseño de la preparación a través del escáner y el software de diseño del sistema EVEREST (CAD), luego se realizó el fresado (CAM) del bloque de Zirconia Everest ZS (Presinterizadas) y la sinterización posterior de la cofia de zirconia, la cual fue evaluada para verificar el adecuado asentamiento de la misma (clínica y radiográficamente) (Figuras 14 y 15). Se realizó el registro clínico del color respectivo para la aplicación posterior de la cerámica de revestimiento (Color 2M2-Vita VM9). Previa a la etapa de cementación definitiva de la restauración coronaria, se realizó la instalación definitiva del pilar protésico personalizado, con un torque de $20 \mathrm{~N} / \mathrm{cm}$ del tornillo protésico. La cementación de la restauración definitiva fue realizada con cementos de ionómero de vidrio modificado con resina (Fuji tipo II). (Figura 16).

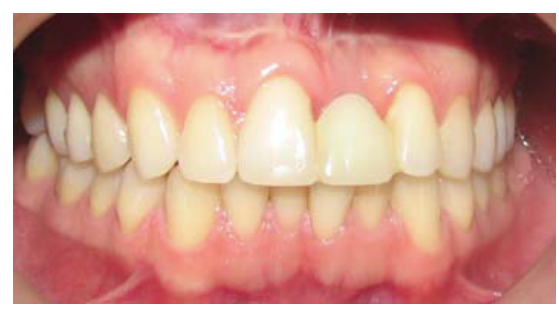

Figura 1. Provisionalización con diente de stock, posterior a la extracción de la pieza dental 2.1

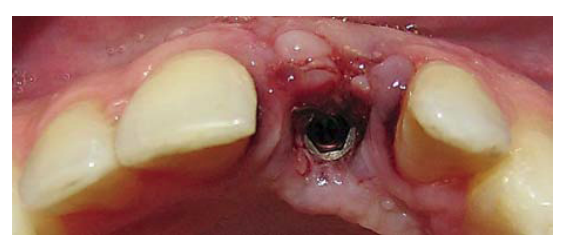

Figura 2. Exposición del implante pza. 2.1, posterior al injerto de tejido conectivo.

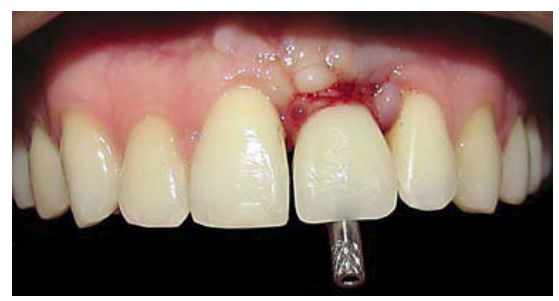

Figura 3. Inicio de etapa de manejo de tejidos blandos con pilar provisional de Ti (pilar UCLA Temporary Sleeve, Hexed SDI 43110). 


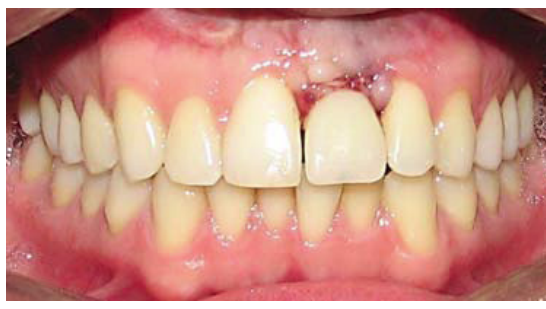

Fig 4. Manejo de tejidos blandos a los 15 días.

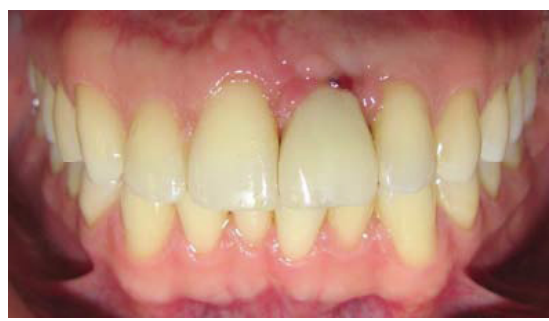

Fig 5. Manejo de tejidos blandos a los 30 días.

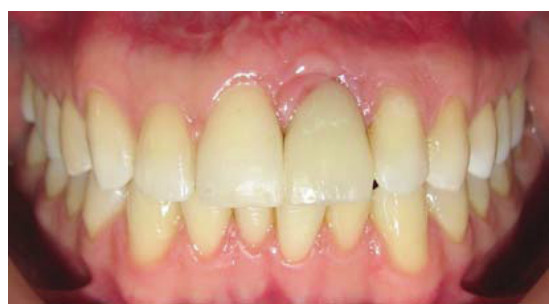

Fig 6. Manejo de tejidos blandos a los 90 días.

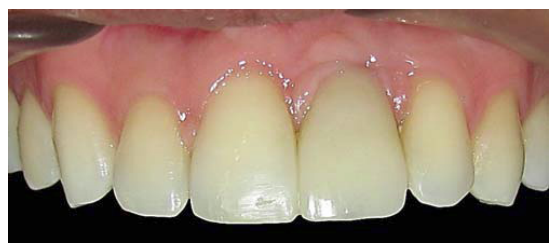

Fig 7. Manejo de tejidos blandos a los 180 días.

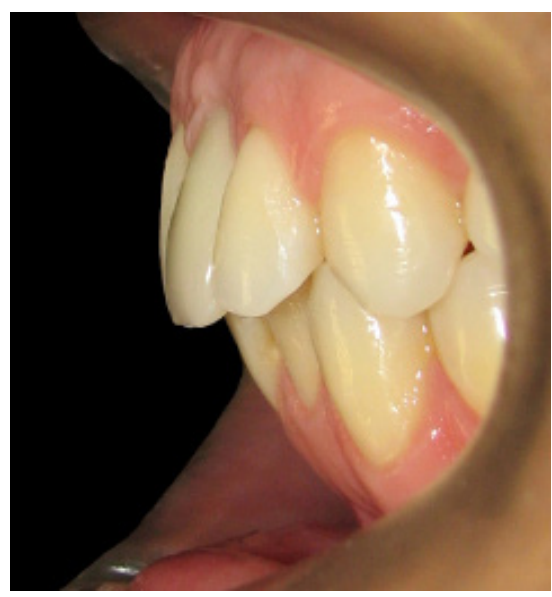

Fig 8. Vista de perfil de emergencia del provisional.

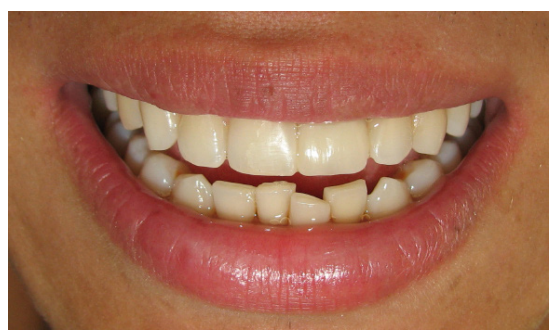

Fig 9. Vista frontal de restauración provisional

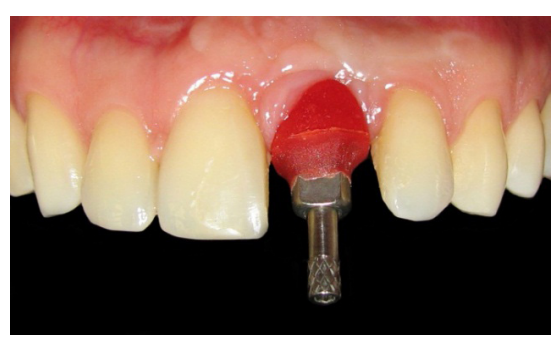

Fig 9. Impresión definitiva personalizada (perfil deemergencia).

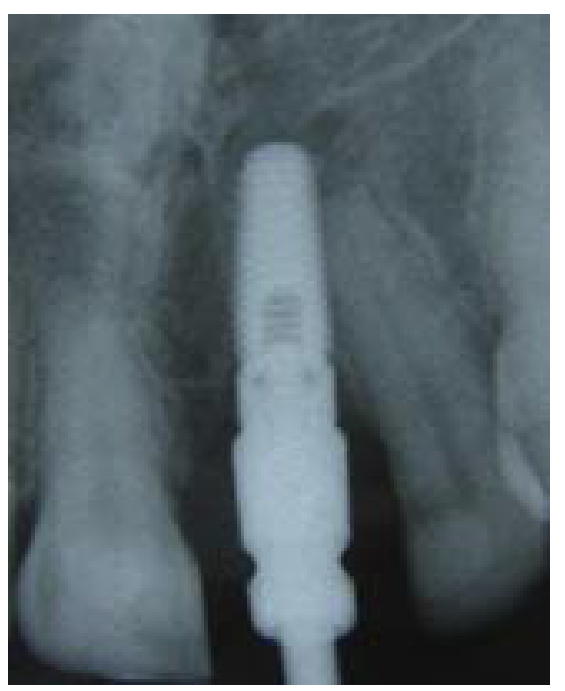

Fig 10. Control Rx.
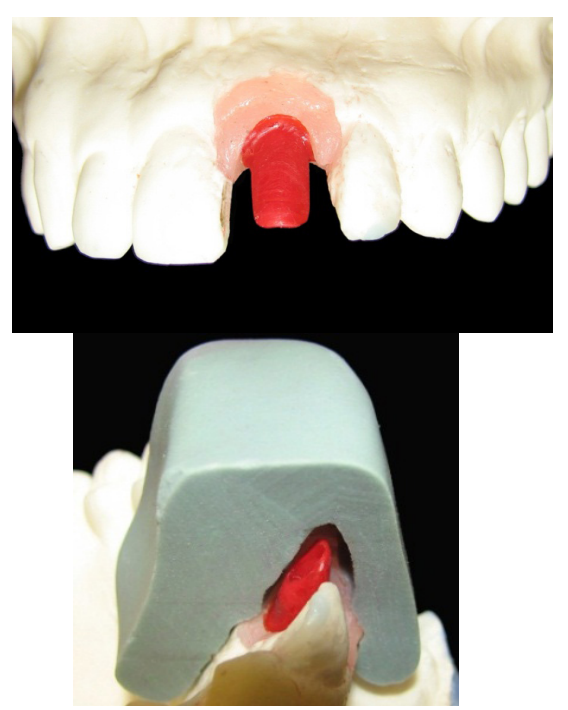

Fig 11. Confección en patrón de duralay del abutment personalizado.
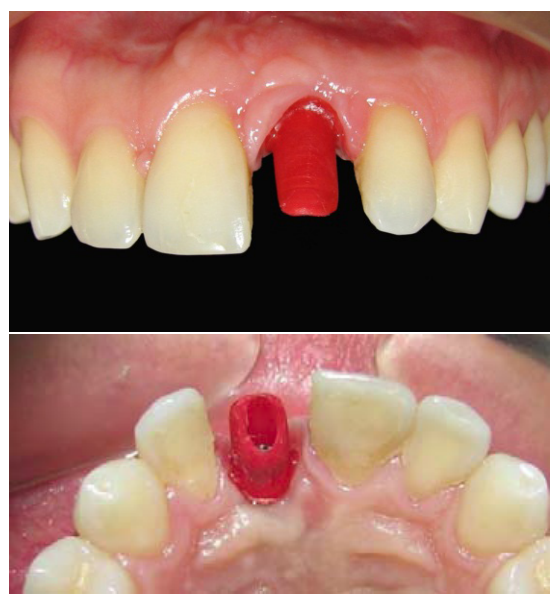

Fig 12. Prueba de pilar UCLA de base metálica de oro-paladio con patrón de duralay y perfil de emergencia según provisional (en boca).

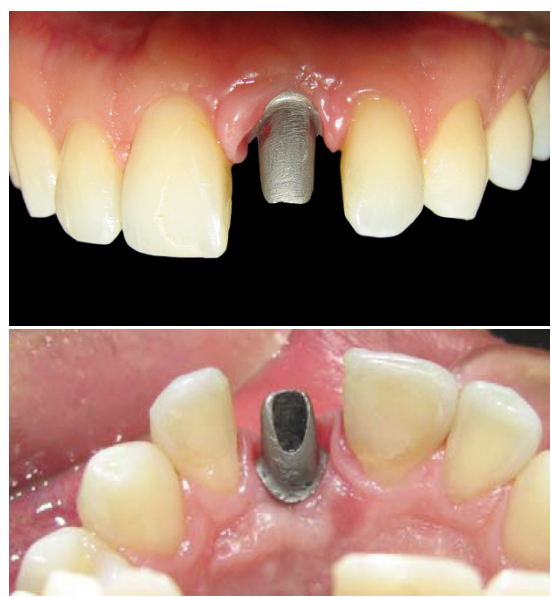

Fig 13. Prueba de pilar anatómico personalizado UCLA de aleación oro- paladio con perfil de emergencia según provisional (en boca).

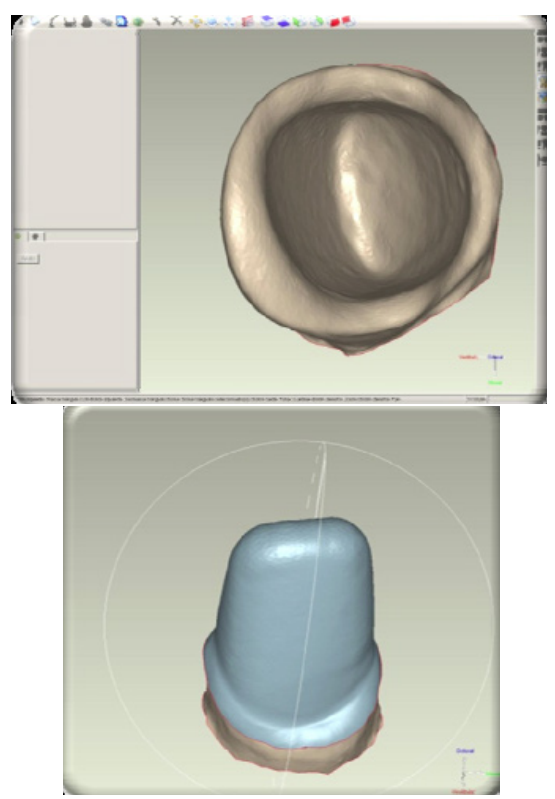

Fig 13. Escaneo y diseño de estructura de zirconia (cofia) en el sistema CAD-CAM Kavo-Everest 


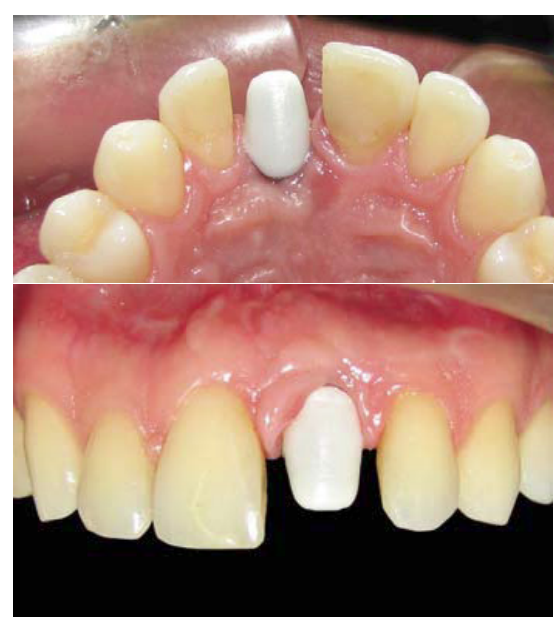

Fig 15. Prueba en boca de cofia de zirconia sistema CAD-CAM Kavo-Everest.

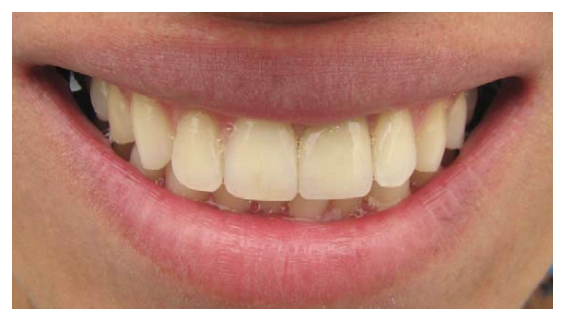

Fig 16. Restauración coronaria finalizada con cerámica de revestimiento Vita VM9. Color $1 \mathrm{M} 2$.

\section{Caso 2: Pilar metal-cerámico}

Paciente masculino de 57 años de edad, acude a la consulta por fractura de pieza 1.1, además presenta antecedentes de fractura de la pieza dental 2.1, con anquilosis de la misma (no presenta movilidad) (Figura 17).

Luego de la etapa de planificación protésico quirúrgica se realiza la extracción de la pieza fracturada (1.1) y la colocación de un implante del sistema Conexão de $5 \mathrm{~mm}$ de $\varnothing$ y $13 \mathrm{~mm}$ de longitud de conexión interna (semi-sumergido). Se colocó una restauración provisional usando el remanente coronario por el período de oseointegración (5 meses) (protocolo de carga diferida) (Figura18).

Posterior al proceso de oseointegración, se realizó la etapa de ma- nejo de tejidos blandos a través de una etapa de provisionalización con un pilar provisional de titanio (cód. 137064), la restauración del frente estético fue con un diente de stock (Ivostar color 310 Chromascop) (Figura19).

A la reevaluación del caso, se planificó una corona libre de metal en la pieza dental 2.1 (objetivo: lograr proporciones adecuadas entre las piezas dentales 1.1 y 2.1). Se realizó el tallado definitivo para una restauración coronaria en la pieza 2.1 (E-Max CAD) y se colocó una restauración provisional. Se verificó que la pieza dental no tenga contacto alguno en estática ni en dinámica. La etapa de manejo de tejidos blandos de los tejidos periimplantarios y estabilización de los mismos, duró 4 meses (Figura19).

Una vez concluida la etapa de provisionalización, se tomaron las impresiones definitivas tanto de la restauración implanto soportada (impresión personalizada en base al perfil de emergencia obtenido en la etapa de manejo de tejidos blandos), como de la restauración sobre el pilar natural (Figuras 20 y 21). Se determinó la necesidad de utilizar un pilar metálico (Ti) con una sobre estructura personalizada de cerámica $(\mathrm{ZrO} 2)$, por los requerimientos estéticos del paciente, y de igual manera para lograr resultados estéticos que se integren adecuadamente con los tejidos bandos circundantes. En el laboratorio se fresó el pilar de Titanio ( $2 \mathrm{~mm}$ Cuff), para que brinde paredes paralelas anti-rotacionales, el cual fue escaneado con el software del sistema CAD-CAM Sirona In-Lab, para diseñar la forma de la estructura cerámica, la cual va a reproducir el perfil de emergencia conseguido con el provisional (Figura 22). Se fresó la estructura en la unidad CAM de Sirona, se realizó la sinterización (7 horas- $1500^{\circ} \mathrm{C}$ ), y luego se probó el asentamiento adecuado de la estructura cerámica sobre el pilar de titanio; se realizó la cementación definitiva de la estructura cerámica sobre el pilar de titanio a través del cemento resinoso Multilink (Ivoclar), previo acondicionamiento de ambos sustratos (metal-cerámica). Se hizo la prueba respectiva en boca del paciente (control visual de la profundidad de la preparación, y control radiográfico del adecuado asentamiento del pilar personalizado sobre el implante) (Figura 23). El pilar personalizado metal-cerámico fue escaneado luego en la unidad CAD de Sirona (escáner In-Lab), para el diseño y posterior fresado de las estructuras de cerámica EMax CAD (MO3) (según anatomía previamente diseñada en el CAD). Se probó las unidades cerámicas pre-cristalizadas para verificar el asentamiento adecuado respectivo, y luego de la etapa de cristalización (35 minutos a $850^{\circ} \mathrm{C}$ ), se individualizó cada unidad coronaria para dar los efectos ópticos adecuados según cada caso. La cementación definitiva fue realizada con el cemento resinoso Variolink II, previo acondicionamiento de ambos sustratos, tanto de las coronas de disilicato de litio (E-max CAD), como del substrato dentinario y del pilar de dióxido de zirconio, se colocó gel de glicerina en los márgenes para evitar la influencia del oxigeno en la polimerización final del cemento resinoso (Figuras 24,25,26,27,28 y 29). 


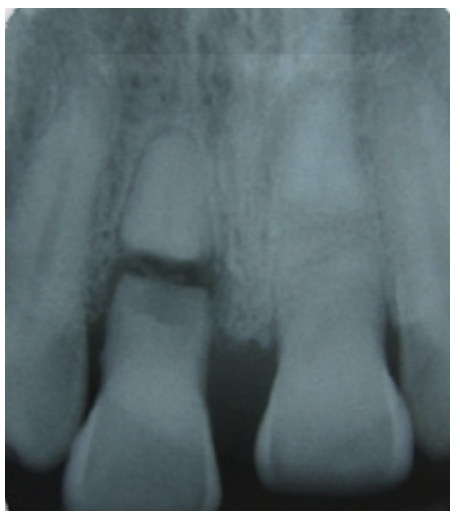

Fig 17. Evidencia radiográfica de fractura en pieza 1.1 .

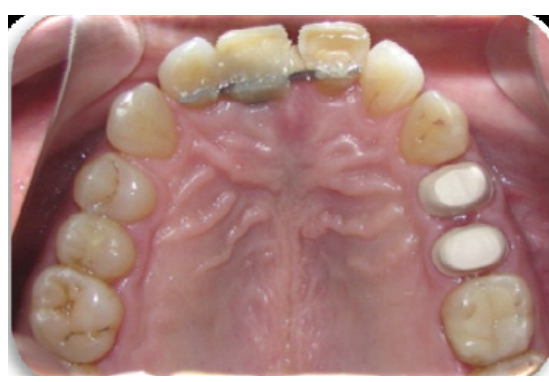

Fig 18. Colocación de implante semi sumergido y provisionalización con diente de stock.

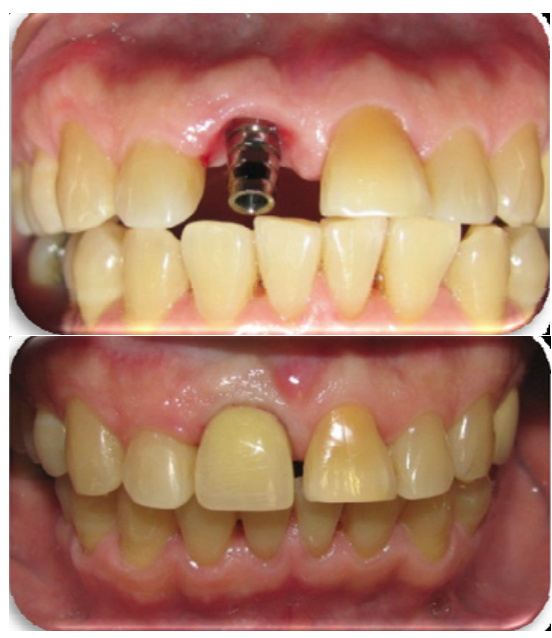

Fig 19. Prueba del pilar provisional y restauración provisional finalizada de implante 1.1 . Se evidencia la necesidad de restaurar la pieza 2.1 para devolver condiciones ideales de proporción tanto en altura como en ancho. El tiempo de manejo de tejidos blandos fue de 4 meses.

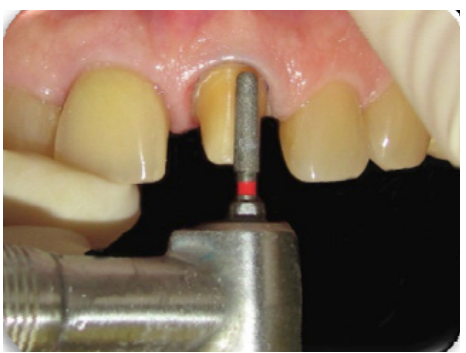

Fig 20. Tallado definitivo de pieza 2.1 para restauración coronaria E-MAX CAD.

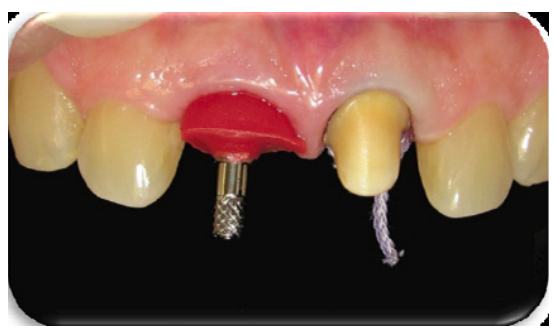

Fig 21. Impresiones definitivas de implante pieza 1.1 y pieza natural 2.1

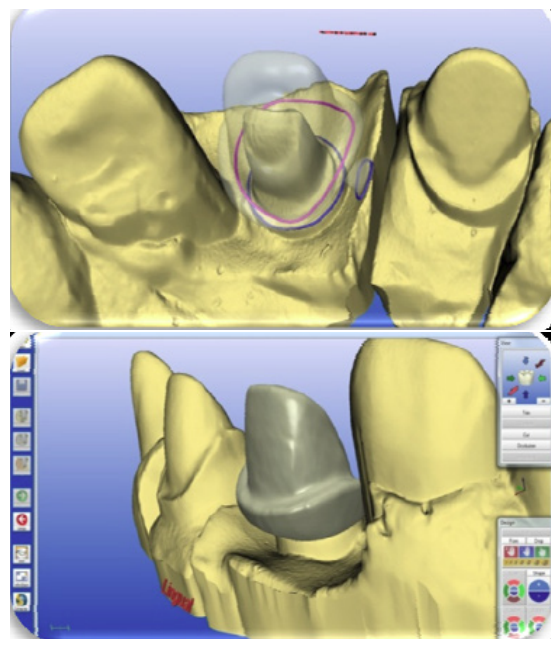

Fig 22. Escaneo, diseño y fresado de estructura personalizada de zirconia sobre el pilar de Ti de implante pieza 1.1 (Sistema CAD-CAM Sirona).

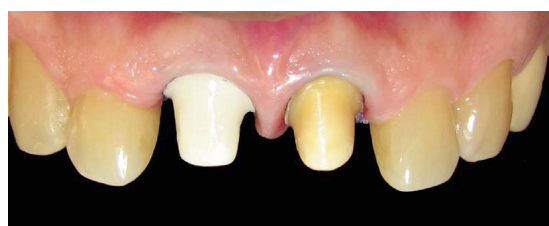

Fig 23. Prueba de asentamiento de pilar personalizado pieza 1.1. Se evidencia el adecuado comportamiento óptico del pilar personalizado metal cerámica con los tejidos blandos circundantes. Impresión definitivas para las restauraciones coronarias de piezas 1.1 y 2.1 (E-Max CAD).

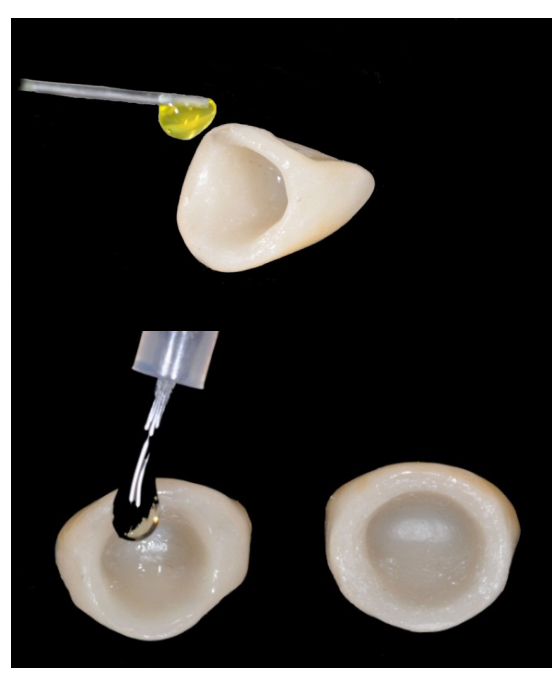

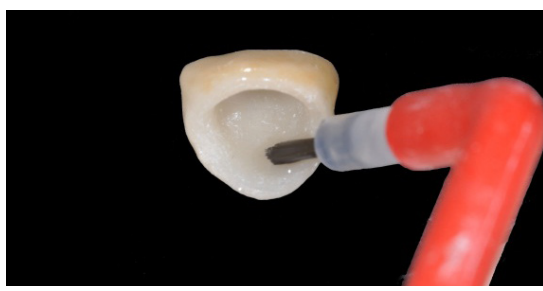

Fig 24. Una vez verificada la oclusión y el sellado marginal de las restauraciones coronarias, se procede al acondicionamiento del substrato cerámico, para realizar una cementación adhesiva (grabado con ác, fluorhídrico al 9\% por 20 seg., lavado con ultrasonido por $5 \mathrm{~min}$., secado, aplicación de silano, y del adhesivo correspondiente (Heliobond-Variolink II).

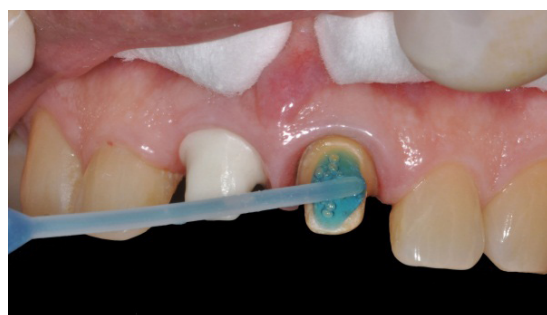

Fig 25. Acondicionamiento del substrato dentinario con ác. fosfórico al $37 \%$ por $15 \mathrm{seg}$., lavado y secado.

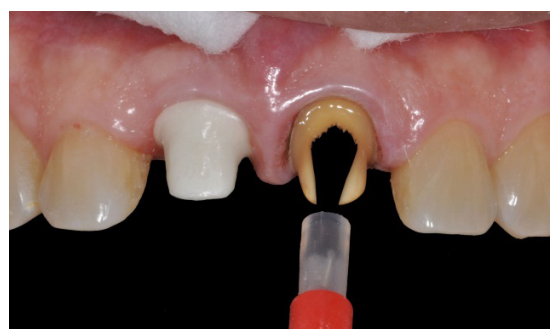

Fig 26. Aplicación del adhesivo Heliobond (Variolink II).

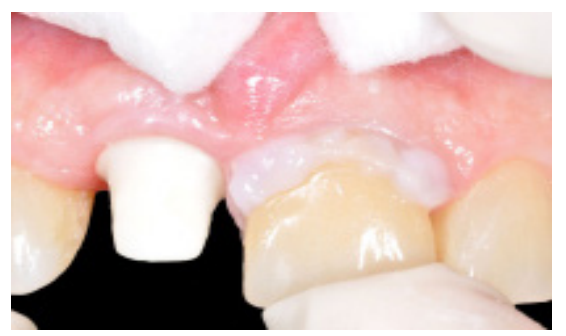

Fig 27. Cementación de restauración coronaria pza. 2.1 con cemento Variolink II. Aplicación de oxyguard para evitar la inhibición de la polimerización (oxigeno) a nivel marginal.

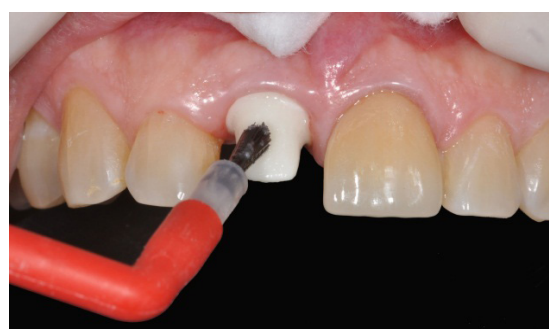

Fig 28. Tratamiento previo de substrato cerámico (zirconia) con primer metálico para zirconia. (Metal / Zirconia Primer). 


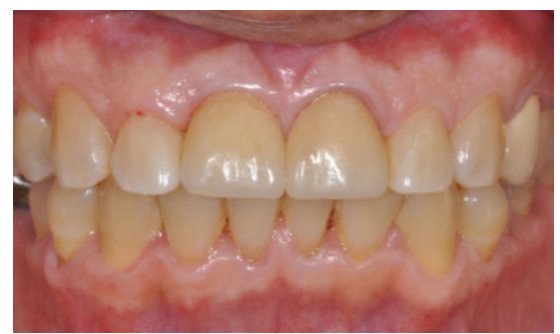

Fig 29. Restauraciones coronarias libres de metal del sistema cerámico E-MAX CAD cementadas en implante 1.1, y pieza dental 2.1. Se observa la adecuada integración de las restauraciones con los tejidos blandos y las piezas dentales contiguas.

\section{Caso 3: Pilar metal-cerámico}

Paciente femenina de 27 años de edad, acude a la consulta por fractura de pieza 1.1 (Figura 30). Se realiza una etapa de planificación protésico quirúrgica, posterior a la cual se realiza la extracción atraumática de la pieza fracturada (1.1) (Figura 31) y se colocó un implante dental (Torque de inserción de $40 \mathrm{~N} / \mathrm{cm}$ ) del sistema Conexão (Master Conect Cónico) de $4.3 \varnothing$ x 15,0 mmde longitud (conexión interna) (Figura 32); se realizó un protocolo de carga inmediata (corona provisional) con el uso de un diente de stock previamente seleccionado de acuerdo al color y proporciones dentarias en relación a la pieza contigua (Ivostar color 130 Chromascop), el cual fue adherido a un pilar provisional de titanio (cód. 137054) (Figuras 33 y 34). Se verificó que la pieza dental no tenga contacto alguno en estática ni en dinámica. La etapa de manejo de tejidos blandos y estabilización de los mismos, duró 6 meses (Figura $35,36,37$ y 38 ).

Una vez concluida la etapa de provisionalización, se tomaron las impresiones definitiva de la restauración implanto soportada (impresión personalizada en base al perfil de emergencia obtenido en la etapa de manejo de tejidos blandos) (Figura 39). Se determinó la necesidad de utilizar un pilar metálico (Ti) con una sobre estructura personalizada de dióxido de zirconio $(\mathrm{ZrO} 2)$ (Y-TZP), en primer lugar porque la paciente presenta un biotipo periodontal delgado y evitar el probable compromiso estético por la traslucidez del tejido en relación a un pilar metálico de titanio convencional, en segundo lugar por los requerimientos estéticos del paciente, y de igual forma lograr resultados estéticos que se integren adecuadamente con los tejidos bandos circundantes. En el laboratorio se fresó el pilar de Titanio ( $2 \mathrm{~mm}$ Cuff), para que brinde paredes paralelas antirotacionales, el cual fue escaneado con el software del sistema CADCAM Sirona In-Lab, para diseñar la forma de la estructura cerámica, la cual va a reproducir el perfil de emergencia conseguido con el provisional. Se fresó la estructura cerámica de dióxido de zirconio (presinterizada) en la unidad CAM de Sirona, luego se realizó la sinterización $\left(7\right.$ horas $\left.-1500^{\circ} \mathrm{C}\right)$, y se probó el asentamiento adecuado de la estructura cerámica sobre el pilar de titanio. Se realizó la cementación definitiva de la estructura cerámica sobre el pilar de titanio a través del cemento resinoso Multilink (Ivoclar), previo acondicionamiento de ambos sustratos (metal-cerámica). Se hizo la prueba respectiva en boca del paciente (control visual de la profundidad de la preparación, y control radiográfico del adecuado asentamiento del pilar personalizado sobre el implante) (Figuras40 y 41). El pilar personalizado metalcerámico fue escaneado luego en la unidad CAD de Sirona (escáner In-Lab), para el diseño y posterior fresado de las estructuras de cerámica E-Max CAD (MO3) (según anatomía previamente diseñada en el CAD). Se probó la unidad cerá- mica pre-cristalizada para verificar el adecuado asentamiento (Figura 42), luego de la etapa de cristalización $\left(35\right.$ minutos a $850^{\circ} \mathrm{C}$ ), se realizó la técnica de cut-back para dar los efectos ópticos adecuados según sea el caso. La cementación definitiva fue realizada con el cemento resinoso Variolink II, (previo acondicionamiento de los sustratos)( figura 43).

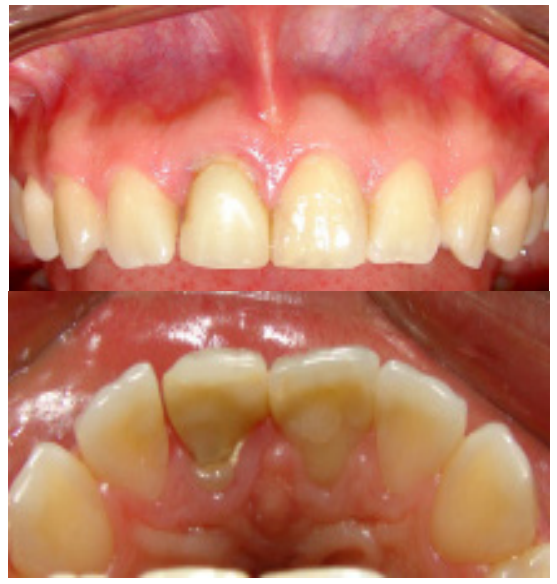

Fig 30. Vistas frontal y palatina de la fractura a nivel de la pieza dental 1.1

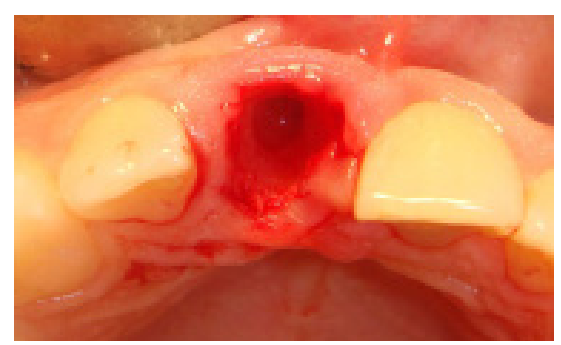

Fig 31. Extracción atraumática de la pieza dental 1.1

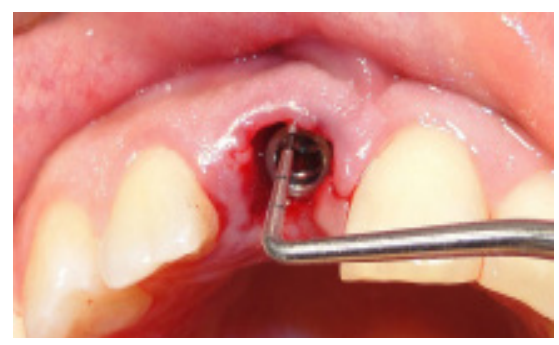

Fig 32. Colocación del implante en óptima posición 3D.

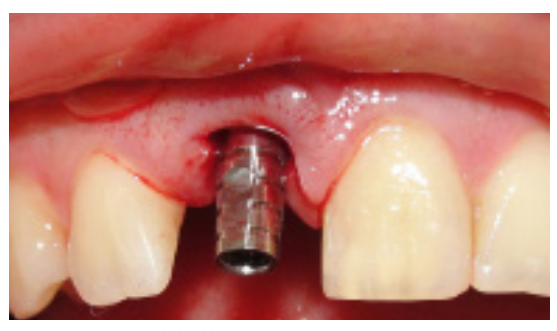

Fig 33. Prueba del pilar provisional sobre el implante 1.1 


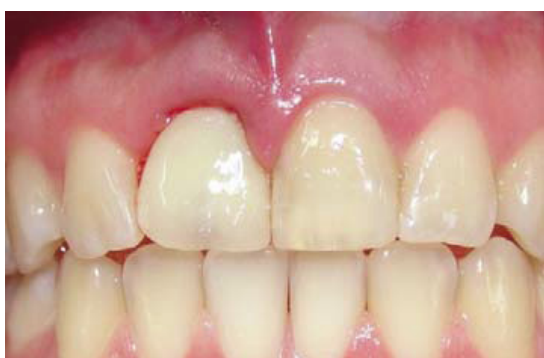

Fig 34. Instalación de provisional sobre el implante 1.1, para posterior etapa de manejo de tejidos blandos.

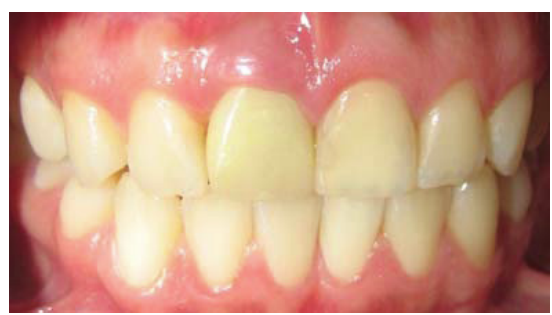

Fig 35. Primera etapa de manejo de tejidos blandos a los 30 días de instalación inicial del provisional.

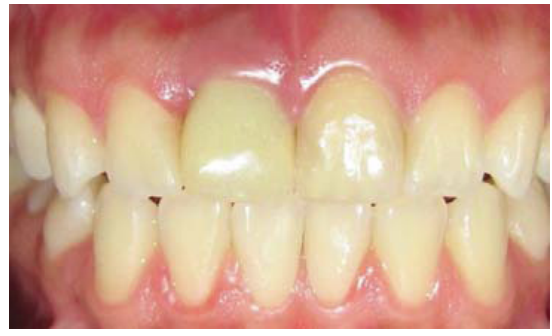

Fig 36. Segunda etapa de manejo de tejidos blandos a los 60 días de la instalación del provisional.

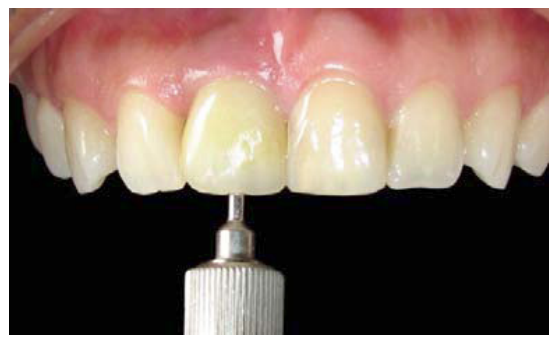

Fig 37. Tercera etapa de manejo de tejidos blandos a los 90 días de instalación inicial del provisional.

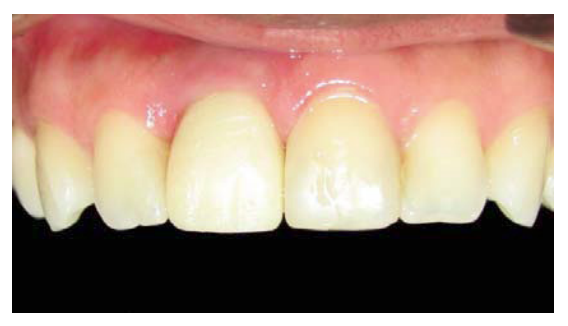

Fig 38. Cuarta etapa de manejo de tejidos blandos a los 120 días de instalación inicial del provisional.

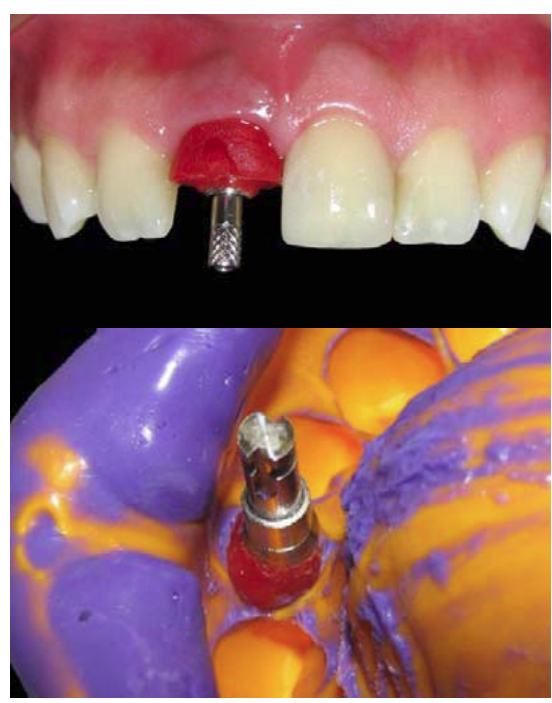

Fig 39. Impresión definitiva de implante 1.1 (transfer personalizado).

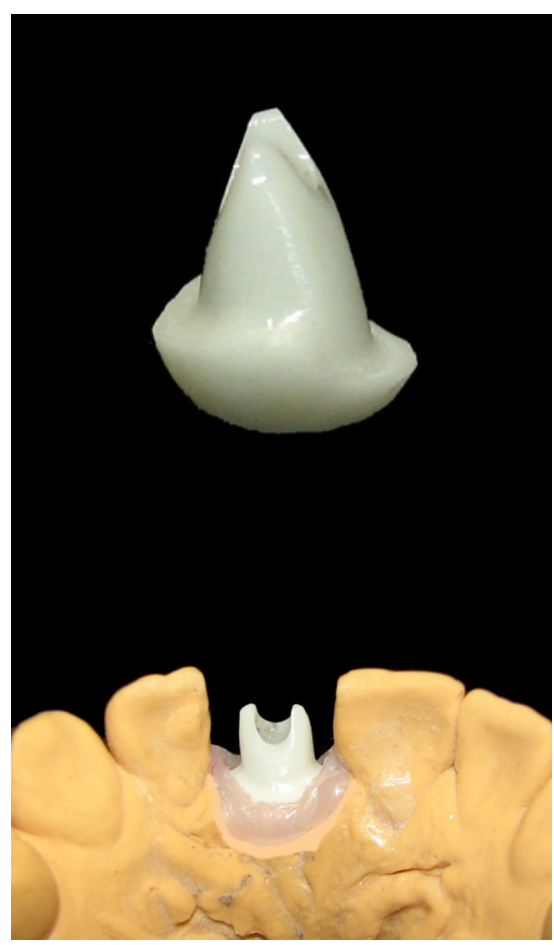

Fig 40. Estructura personalizada de zirconia.

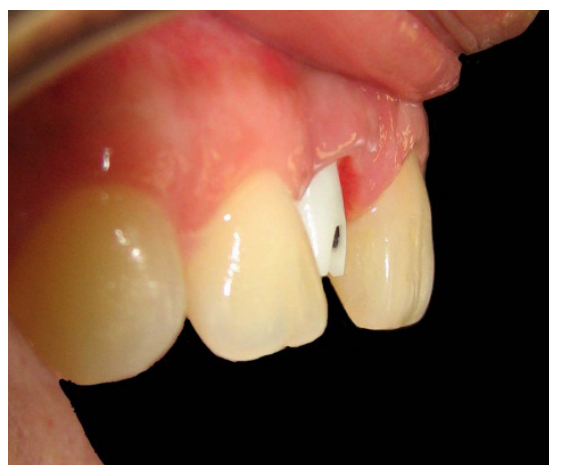

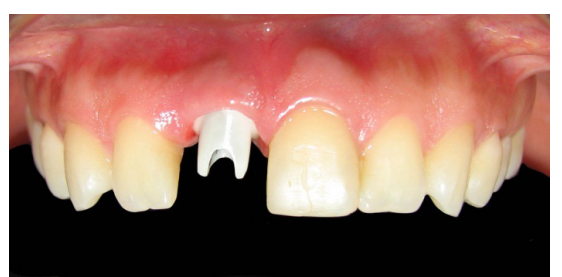

Fig 41. Prueba de pilar anatómico personalizado de zirconia en boca. Vista frontal y de perfil.

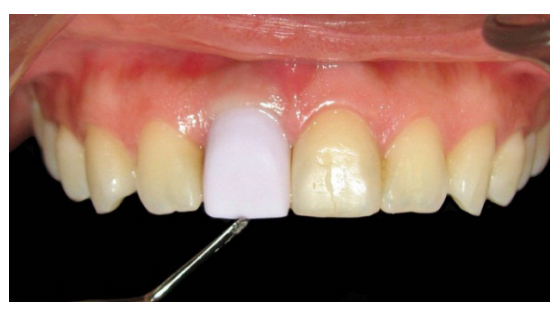

Fig 42. Estructura de corona E-MAX CAD pre cristalizada.

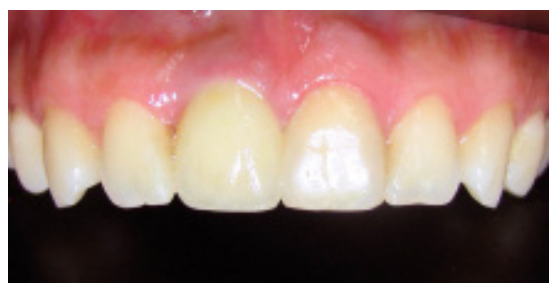

Fig 43. Corona E-MAX CAD cementada en boca.

\section{Conclusiones}

Los criterios de decisión que deben ser considerados con respecto al tipo de material del abutment para las restauraciones unitarias de implantes en el sector anterior deben ser biotipo y espesor de la gingiva, color y matiz de los dientes contiguos, región visible o no visible (anterior o posterior) de la arcada, expectativas estéticas del paciente y costos. En las regiones posteriores, los abutments prefabricados de titanio son los indicados (6). Factores críticos para la elección de uno u otro material son su alta resistencia, procedimientos de laboratorio simples, y bajo costo. Además el paciente debe ser informado de que una probable decoloración puede ser observada en el margen gingival de la corona si se utiliza abutments metálicos en la región anterior dependiendo de las condiciones clínicas del biotipo 
periodontal de cada paciente. En algunas situaciones clínicas debido a las dificultades en el retiro de los agentes cementantes se recomienda el uso de restauraciones atornilladas sólo en regiones que presenten una mucosa baja. En el área anterior, sólo se pueden recomendar abutments prefabricados en casos de mucosa baja y con baja demanda estética.

Considerando los criterios listados anteriormente, sobre la posición 3D de la colocación de un dispositivo implantario, una restauración personalizada metálica con el uso de un abutment de tipo UCLA puede ser considerado y puede ser enmascarado adecuadamente con una restauración libre de metal (núcleos cerámicos de infraestructura: sistema In-Ceram, Zirconia).

En situaciones clínicas donde las demandas estéticas del paciente son prioritarias, un injerto de tejido conectivo es recomendado previo a la instalación del abutment, para mejorar las condiciones clínicas de los tejidos blandos y tener una mejor previsibilidad en el tiempo de los mismos.

El uso de abutments personalizados de zirconia son recomendados en casos de alta estética y en los que se requiere una adecuada interrelación entre el material de reconstrucción y los tejidos blandos peri-implantarios circundantes.

Si bien es cierto, como se mencionó, que una de las complicaciones que se podrían presentar al momento de la cementación de una corona de tipo cementada es la dificultad en el retiro del cemento, el uso de los pilares anatómicos personalizados provee la posibi- lidad de determinar una adecuada profundidad del margen de la restauración, facilitando la adecuada remoción de los excesos del agente cementante y evitando así complicaciones en los tejidos blandos circundantes. De igual manera brinda la posibilidad de dar un adecuado soporte al tejido blando peri-implantario a través de la determinación de un adecuado perfil de emergencia de las futuras restauraciones coronarias.

\section{Referencias Bibliográficas}

1. Alfarsi MA, Mehmet Okutan H, Bickel M. CAD/CAM to fabricate ceramic implant abutments and crowns: a preliminary in vitro study. Australian Dental Journal 2009; 54: 12-16.

2. Funato A, Salama MA, Ishikawa T, Garber D, Salama H. Timing, positioning, and sequential staging in esthetic implant therapy: A four-dimensional perspective. Int J Periodontics Restorative Dent. 2007; 27: 313-323.

3.Fürhauser R, Florescu D, Benesch T, Haas R, Mailath G, Watzek G. Evaluation of soft tissue around single-tooth implant crowns: the pink esthetic score. Clin Oral Impl Res. 2005; 16(6): 639-644.

4. Gershenfeld L. Objective criteria of aesthetic implant success: The pink and white aesthetic scores. Australasian Dental Practice. 2009; 156-159.

5.Kohal RJ, Att W, Bächle M, Butz F. Ceramic abutments and ceramic oral implants. An update. Periodontology. 2008; 47: 224243.

6. Bindl A. Enhanced light transmission. Australasian Dental Practice. 2010;21(5): 184-186.

7.Strub JR, Rekow ED, Witkowski
S. Computer-aided design and fabrication of dental restorations: current systems and future possibilities. J Am Dent Assoc. 2006; 137:1289-1296.

8.Sailer I, Zembic A, Jung RE, Hämmerle $\mathrm{CH}$, Mattiola A. Single-tooth implant reconstructions: esthetic factors influencing the decision between titanium and zirconia abutments in anterior regions. Eur J Esthet Dent. 2007; 2(3): 296-310. 